\title{
Recovery in ERG gene expression with biventricular pacing in a rabbit model of myocardial infarction
}

This article was published in the following Dove Press journal:

Research Reports in Clinical Cardiology

20 May 2013

Number of times this article has been viewed

\author{
Samir Saba* \\ Haider Mehdi* \\ William Barrington \\ Fardeen Mehdi \\ Zahid Islam \\ Barry London \\ Cardiovascular Institute of the \\ University of Pittsburgh Medical \\ Center, Pittsburgh, PA, USA \\ *These authors contributed equally \\ to this work
}

Correspondence: Samir Saba

Cardiovascular Institute, University of Pittsburgh Medical Center, 200 Lothrop Street, B535 PUH, Pittsburgh, PA 15213, USA

Tel + I 4126476272

Fax + I 4126477979

Email sabas@upmc.edu
Background: Improved clinical and echocardiographic parameters have been documented with biventricular (BIV) pacing in patients after myocardial infarction (MI). We investigated the changes in gene expression in cardiac tissue with BIV pacing, using a rabbit model of MI.

Method: New Zealand White rabbits were divided into four groups: sham-operated controls, MI with no pacing, MI with right ventricle (RV) pacing (MI + RV), and MI with BIV pacing $(\mathrm{MI}+\mathrm{BIV})$. Pacing was initiated 1-2 weeks after the coronary ligation. At 5 weeks, the hearts were excised. The tissue extracted from the left ventricle (LV) and RV underwent analysis for protein and messenger ribonucleic acid (mRNA) levels.

Results: The ether-a-go-go-related gene (ERG) protein levels recovered from the base of the LV away from the MI area were two- to threefold lower in the MI and the MI + RV compared with the $\mathrm{MI}+\mathrm{BIV}$ groups $(P=0.07)$. The ERG protein levels were similar between the MI + BIV and the control groups. However, the RNA levels were comparable between the four study groups, suggesting that a posttranscriptional mechanism accounted for the difference in protein levels.

Conclusion: In this rabbit model of MI, we demonstrated a recovery in ERG protein levels with BIV pacing, after MI. This recovery may underlie some of the benefits seen with BIV pacing in ischemic cardiomyopathy.

Keywords: heart failure, biventricular pacing, cardiac reverse remodeling, ether-a-go-gorelated gene

Cardiac resynchronization therapy, also known as biventricular (BIV) pacing, is a modality used in the treatment of advanced heart failure ${ }^{1-3}$ In patients with ventricular conduction abnormalities who continue to suffer from severe heart failure symptoms (New York Heart Association Class III-IV) ${ }^{1-3}$ despite optimal pharmacological therapy, BIV pacing can improve left ventricle (LV) muscle function and induce reverse mechanical remodeling, manifested as smaller LV end-systolic and end-diastolic dimensions. Clinical trials have also demonstrated the benefits of BIV pacing in improving the endpoints of death and hospitalization. ${ }^{2,3}$

Despite this, little is known about the regional, cellular, and molecular mechanisms responsible for the benefits seen with BIV pacing. In a previous study ${ }^{4}$ we demonstrated the prevention of remodeling with BIV pacing initiated at the time of myocardial infarction (MI), manifested as the preservation of LV function and dimensions as well as preservation of the protein levels of the ether-a-go-go-related gene (ERG) protein. In this current study, we investigated whether changes in the myocardial ERG protein levels can be demonstrated when the BIV pacing is initiated 1-2 weeks after the MI, which better simulates the clinical scenario observed with heart failure patients. 


\section{Methods}

\section{Study design}

The study protocol was approved by the University of Pittsburgh Institutional Animal Care and Use Committee. New Zealand White rabbits were housed at the animal facility in individual cages and allowed free access to food pellets, hay, and water. The rabbits $(\mathrm{n}=12$; weight $=3.0-5.0 \mathrm{~kg})$ were divided into four study groups: (1) sham-operated controls $(C)(n=3)$, in which the rabbits underwent pericardial stripping only; (2) MI with no pacing (MI group) $(\mathrm{n}=3)$, in which the rabbits underwent pericardial stripping and coronary ligation but no pacing; (3) MI with right ventricle (RV) pacing ( $\mathrm{MI}+\mathrm{RV}$ group) $(\mathrm{n}=3)$, in which the rabbits underwent pericardial stripping, coronary ligation, and RV pacing with the intent of creating ventricular dyssynchrony; and (4) MI with BIV pacing (MI + BV group) $(n=3)$, in which the rabbits underwent pericardial stripping, coronary ligation, and BIV pacing. Rabbits in the pacing groups were continuously paced at a rate of 270 beats per minute for 3-4 weeks beginning 1-2 weeks after coronary ligation and pacemaker implantation, depending on the rate of animal recovery after surgery. At the end of this period, they were sacrificed, and their hearts were excised. Cardiac tissue was collected from the base of the LV away from the MI site as well as from the RV. Tissue analysis was performed as described below.

\section{Surgical preparation of the rabbits}

The rabbits were anesthetized using an intramuscular injection of a mixture of ketamine $(35 \mathrm{mg} / \mathrm{kg})$ and xylazine $(5 \mathrm{mg} / \mathrm{kg})$. A 22 gauge intravenous catheter was inserted into the marginal ear vein for venous access. An arterial line was inserted into the middle ear artery, for continuous hemodynamic monitoring. The rabbits were intubated with an endotracheal tube (3.0 mm ID) and mechanically ventilated (rate $40 / \mathrm{min}$, tidal volume $15 \mathrm{~mL}$ ) using room air enriched with oxygen. Isoflurane anesthesia $(2.0 \%-2.5 \%)$ was delivered, to maintain general anesthesia during surgery. The rabbits were placed on a water blanket adjusted to $38^{\circ} \mathrm{C}$. A pulse oxymeter was placed on the rabbit's tongue for continuous monitoring of oxygen saturation.

The chest was opened through the fourth left intercostal space. The heart was exposed through an incision of the pericardium and explored. The MI was induced by ligating the posterolateral branch of the left coronary system, using a 5-0 Prolene ${ }^{\mathrm{TM}}$ suture (Ethicon Inc, Somerville, NJ, USA) at a level midway between the atrioventricular groove and the apex. Successful ligation was confirmed by the presence of myocardial cyanosis, with bulging and ST-segment changes in the amplified electrocardiogram (ECG) signal. These changes in the surface ECG were previously described in detail by our group. ${ }^{4}$ Lidocaine $(1-4 \mathrm{mg} / \mathrm{kg}$ ) was administered pre- and postligation as an antiarrhythmic agent, and a prophylactic antibiotic (Ancef $100 \mathrm{mg}$ intravenous [IV]) was administered before and after surgery.

\section{Pacemaker implantation}

Pacemaker leads were sutured to the epicardial surface of both the RV and LV, or on the RV only, depending on the study group of the rabbit. For RV pacing, the lead was sutured to the RV free wall, close to the apex. For LV pacing, the lead was sutured to the LV free wall, close to the base of the heart. The leads (model 4965 and model 4968; Medtronic, Inc, Minneapolis, MN, USA) were connected to a permanent pacemaker configured to pace the RV alone or both the RV and LV simultaneously. In case of BIV pacing, a Y-connector (model 2872; Medtronic) was used to pace the RV and LV simultaneously from a single-chamber pacemaker (Kappa KSR403; Medtronic), programmed through research software (Medtronic Inc) to pace at a fast rate of 270 beats per minute (slightly above the encaged rabbits' ambulatory heart rate). The pacemaker and leads were placed in a subcutaneous pocket in the abdominal area of the rabbit.

\section{Cellular and molecular analysis of cardiac tissue}

The hearts were excised from the rabbits, and $200 \mathrm{mg}$ samples from the base of the LV free wall and the RV free wall were collected. We performed immunoblots (Western blots) using anti-glyceraldehyde 3-phosphate dehydrogenase (GAPDH) (Bethyl Laboratories, Inc., Montgomery, TX, USA) anti-Erg (Life Technologies Corp, Carlsbad, CA, USA), anti-KVLQT1 (Alomone Labs, Jerusalem, Israel), and anti-Kv4.3 (Alomone Labs). As previously described, ${ }^{5}$ crude membrane preparations were isolated, by differential centrifugation, from the same regions of the hearts described above. Channels were solubilized with $1 \%$ sodium dodecyl sulfate (SDS), quantitated (BioRad Laboratories, Hercules, CA, USA), and $\sim 100 \mu \mathrm{g}$ of protein per lane was run on a $12 \%$ SDS-polyacrylamide gel electrophoresis (PAGE) gel, transferred by semidry apparatus, blocked with phosphate-buffered saline (PBS [Cellgro, Manassas, VA, USA])-5\% milk, incubated overnight at $4{ }^{\circ} \mathrm{C}$ with the primary antibody, washed with PBSTween ${ }^{\circledR}$ (Bio-Rad Laboratories, Inc., Hercules, CA, USA), incubated with horseradish peroxidase (HRP)-conjugated 
chicken anti-rabbit $2^{\circ}$ antibody (Amersham Biosciences, Piscataway, NJ, USA), ${ }^{\circledR}$ and quantitated by chemiluminescence (Perkin-Elmer, Waltham, MA, USA). Coomassie Blue (Life Technologies, Grand Island, NY, USA) staining of the SDS-PAGE blots was performed to confirm equal loading. Custom-made polyclonal antibodies to rabbit ERG, directed against the peptide RQRKRKLSFRRRTD (AA 885-898), were generated in the chicken (affinity-purified IgY; Life Technologies). Preimmune IgY, from egg yolk, was used to determine the SERCA2a- and ERG-specific bands.

The autoradiographs were scanned using a Visioneer OneTouch 9220 scanner (Visioneer Inc, Pleasanton, CA, USA) and Scanner and Camera Wizard software (Microsoft Corp, Redmond, WA, USA). The images were digitized for analysis using Quantity One ${ }^{\mathrm{TM}}$ quantitation software (Bio-Rad). Bands of interest were delineated and densitometries were calculated, based on the number of pixels. We performed Western blot analyses on tissue from all rabbits of each of the C, MI, MI + RV, and MI + BIV groups.

\section{Messenger ribonucleic acid (mRNA) levels}

$E R G$ gene expression levels of mRNA levels were determined by fluorescence-based kinetic real-time polymerase chain reaction (RT-PCR) using an Applied Biosystems ABI PRISM $^{\circledR}$ model 7000 (Life Technologies) sequence detection system. Total RNA was isolated using TRIzol ${ }^{\circledR}$ reagent (Life Technologies), as described elsewhere. ${ }^{6,7}$ First, strand complementary deoxyribonucleic acid (cDNA) synthesis was performed with $1 \mu \mathrm{g}$ of RNA and SuperScript ${ }^{\circledR}$ III RNase $H^{-}$Reverse Transcriptase $(200 \mathrm{u} / \mu \mathrm{L})$ (Life Technologies), and the RT-PCR reaction was performed using $1 \mu \mathrm{g}$ of cDNA, ABsolute QPCR SYBR Green ROX Mix (Thermo Fisher Scientific, Waltham, MA, USA), and rabbit-specific ERG forward: 5'-CAGGCACCACGCATCCA-3' and reverse: 5'-CAGTCCCACACAGCCTTGAA-3' ${ }^{\prime}$ primers. ${ }^{6}$ The quantification of the ERG PCR product was performed with ABI PRISM 7000 SDS software v 1.1, and the expression of $E R G$ was compared with the gene expression of $\beta$-actin using rabbit specific forward: $5^{\prime}$-CTGGCTGGCCGCGACCT-3' and reverse: 5'-GAACCGCTCATTGCCAATGGG-3' $\beta$-actin primer pairs with a similar RT-PCR condition as that of $E R G$. The threshold cycle $\left(\mathrm{C}_{\mathrm{T}}\right)$ number at which the detectable fluorescence for the ERG product exceeded the background was compared with the $\beta$-actin product, for each sample.

\section{Data analysis}

Data is presented as mean \pm standard error unless otherwise indicated. Continuous variables were compared by the Fischer's exact test between the various study groups. Bar graphs were constructed for all analyses and visually inspected. A one-sided $P$-value less than $\leq 0.05$ was considered statistically significant and $\leq 0.1$ was considered to indicate a strong trend towards significance. All statistical analyses and graphs were performed on SPSS software $\mathrm{v} 13$ (IBM, Armonk, NY, USA).

\section{Results}

\section{Myocardial infarction}

As previously described, ${ }^{4}$ all myocardial infarctions were ascertained at the time of the coronary ligation of the posterolateral coronary artery, by myocardial blanching and bulging, as well as by ST elevations on the surface ECG (Figure 1). As previously shown, ${ }^{4}$ when the coronary artery is ligated halfway between the LV base and apex, the sizes of the MIs are predictable and comparable between study groups.

\section{Tissue analysis}

Tissues were collected from rabbits in the C, MI, MI + RV, and $\mathrm{MI}+\mathrm{BIV}$ groups from prespecified regions of the $\mathrm{LV}$ and RV, with focus on the normal myocardial zone, away from the infarcted area. Testing the expression of the $\mathrm{K}^{+}$channels responsible for repolarization showed no differences in the expression of Kv4.3 or KVLQT1, between the groups (data not shown).

Compared with the $\mathrm{C}$ group, ERG protein levels, adjusted for nicotinamide adenine dinucleotide phosphate (NAPDH) loading, obtained from tissues collected from the LV base were two to threefold reduced in the MI group and the MI + RV groups compared with both the $\mathrm{MI}+\mathrm{BIV}$ and $\mathrm{C}$ groups $(P=0.07)$ (Figure 2). In fact, ERG levels in the $\mathrm{MI}+\mathrm{BIV}$ group were comparable to those of the $\mathrm{C}$ group $(P=0.49)$. These difference were not seen in the RV free wall tissue, where the mere presence of pacing ( $\mathrm{MI}+\mathrm{RV}$ and $\mathrm{MI}+\mathrm{BIV}$ groups) resulted in a significant increase (greater than threefold) in the ERG protein expression $(P=0.01$ and $P=0.08$, respectively).

\section{mRNA levels}

The levels of mRNA for the ERG gene were not different among the four groups of rabbits (Figure 3), suggesting a posttranscription mechanism was responsible for the reduction in the LV ERG protein levels in the MI and MI + RV groups compared with the $\mathrm{C}$ and $\mathrm{MI}+\mathrm{BIV}$ groups. 


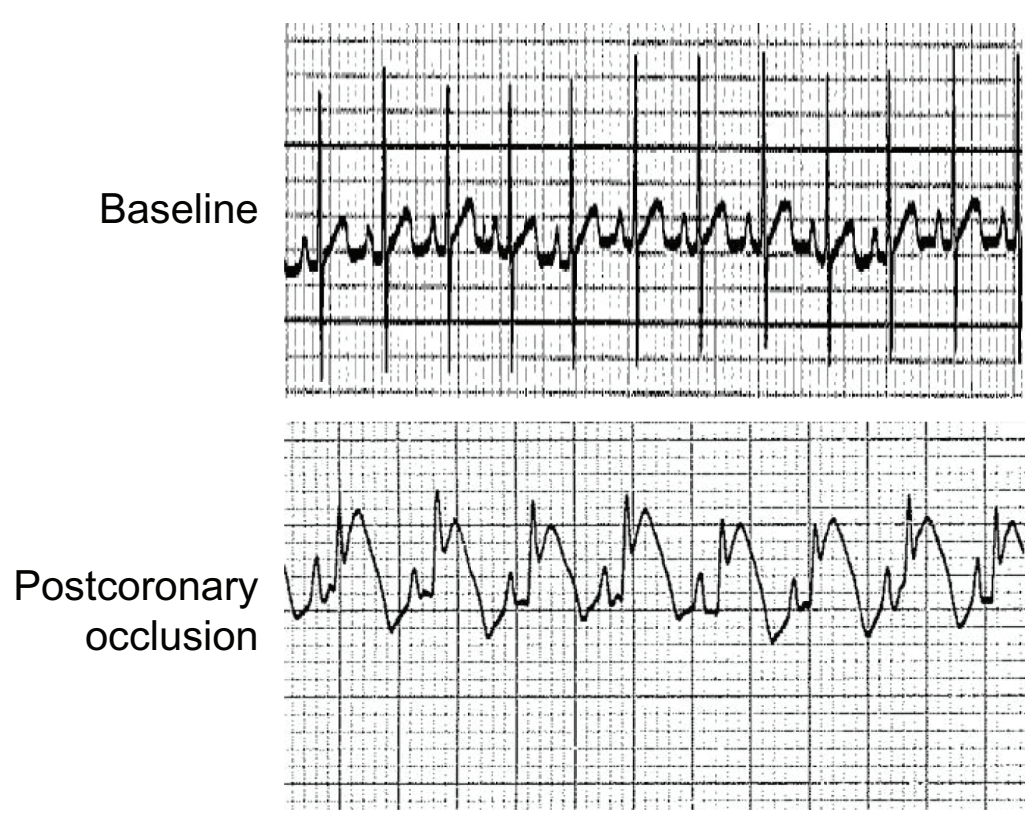

Figure I Surface electrocardiogram lead II demonstrating baseline and postcoronary occlusion tracings in a rabbit included in the MI + BIV study group. Notes: Paper speed $25 \mathrm{~mm} / \mathrm{second}$; resolution $20 \mathrm{~mm} / \mathrm{mV}$. Note the significant ST elevation after coronary occlusion and the loss of R wave amplitude. Abbreviation: $\mathrm{MI}+\mathrm{BIV}$, myocardial infarction plus biventricular pacing.

\section{Discussion}

In this study, we present a rabbit model of MI and pacing, in which we demonstrated the recovery of ERG protein levels with BIV pacing compared with RV pacing or no pacing, to levels comparable with those of $\mathrm{C}$ group rabbits. This was shown in the normal areas of myocardium away from the infarcted LV zone. The mechanism underlying the differences in ERG protein levels must have been posttranscriptional given the identical mRNA levels in the four study groups, possibly related to the translation of mRNA to protein or to other mechanisms, such as protein trafficking to the cell membrane. Our data shows that BIV pacing prevents changes in ERG
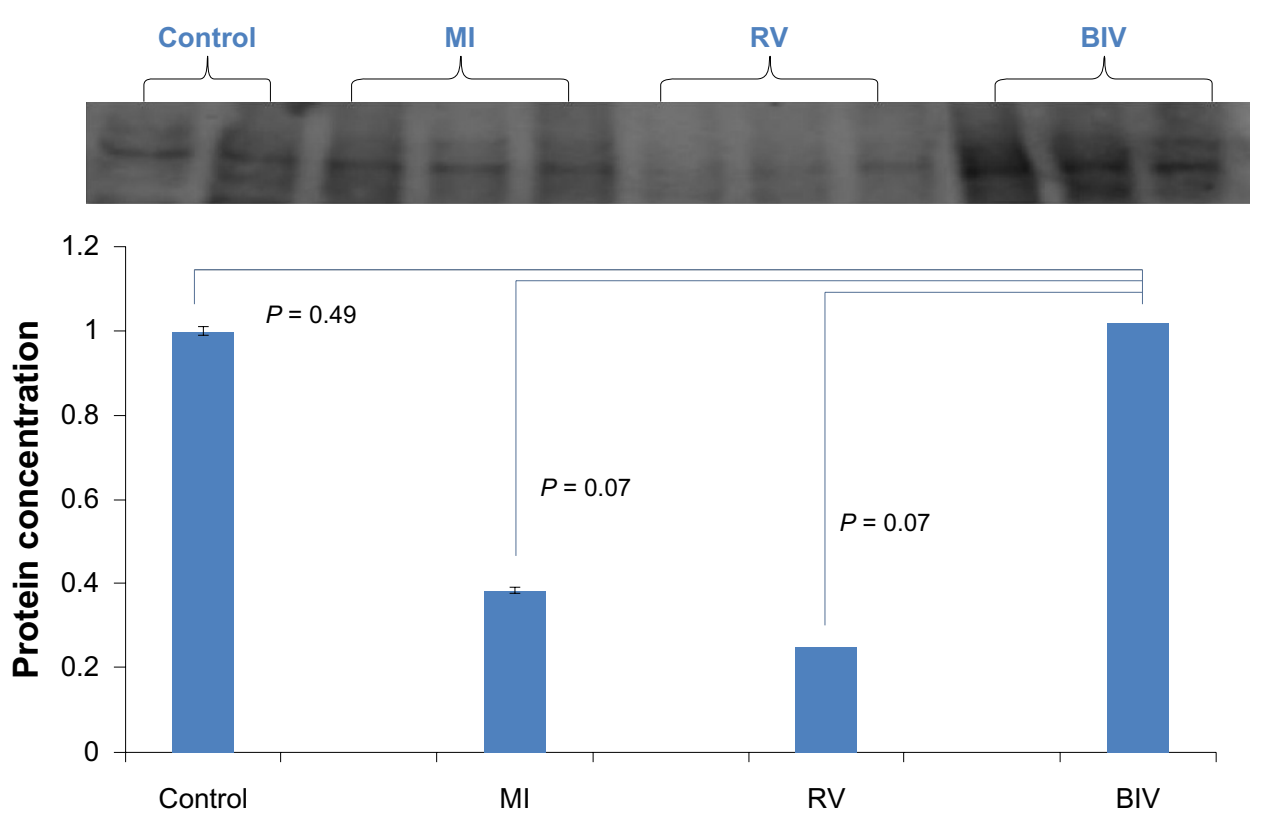

Figure 2 Western blot showing ERG protein levels in two rabbits from the C group and three rabbits from each of the MI, MI + RV, and MI + BIV study groups. Notes: Underneath the Western blot gel, a bar graph represents the levels of ERG protein, corrected for the loading of the gel, with the $45 \mathrm{kD}$ band used as a reference. Note that ERG protein levels are reduced by a factor of two- to threefold in the $\mathrm{MI}(P<0.07)$ and $\mathrm{MI}+\mathrm{RV}(P<0.07)$ groups compared with the control and $\mathrm{MI}+\mathrm{BIV}$ groups. Abbreviations: BIV, biventricular pacing; C, control group; ERG, ether-a-go-go-related gene; MI, myocardial infarction; MI + BIV, myocardial infarction plus biventricular pacing; MI + RV, myocardial infarction plus right ventricular pacing; RV, right ventricular pacing. 


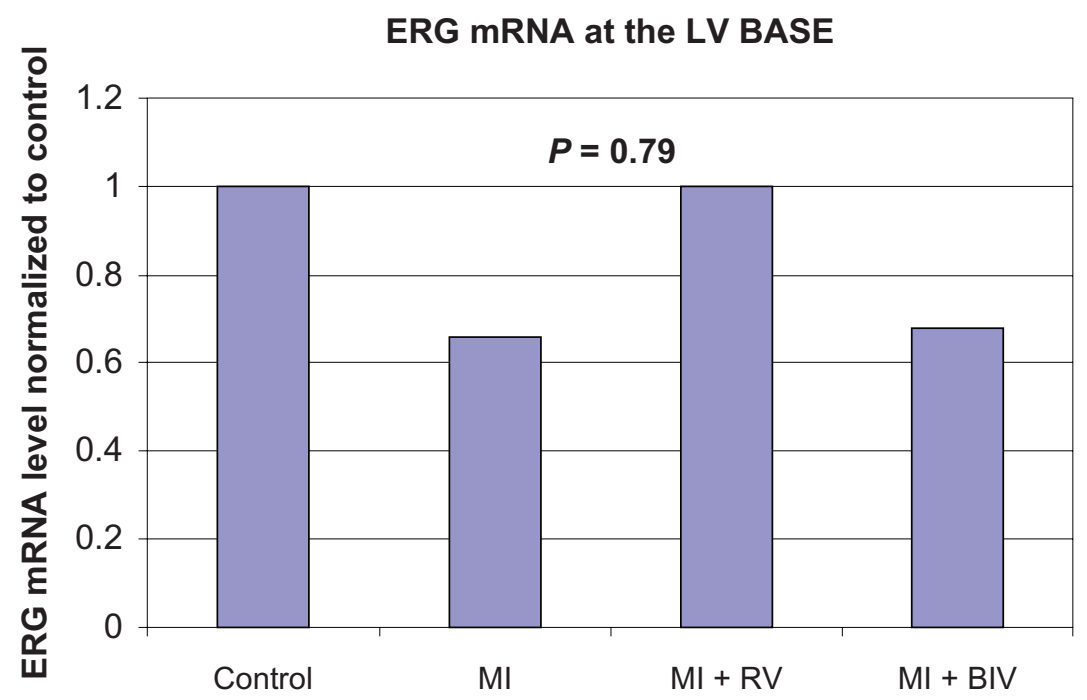

Figure 3 mRNA levels of the ERG gene demonstrating no significant differences among these four groups of rabbits.

Abbreviations: BIV, biventricular pacing; ERG, ether-a-go-go-related gene; LV, left ventricle; MI, myocardial infarction; MI + BIV, myocardial infarction plus biventricular pacing; $M I+R V$, myocardial infarction plus right ventricular pacing; mRNA, messenger ribonucleic acid.

level that are associated with MI, which may underlie some of the mechanisms of benefit of cardiac resynchronization therapy. In keeping with our previous findings, ${ }^{4}$ our present data demonstrate similar changes in the ERG protein levels despite the fact that BIV pacing was not initiated immediately at the time of the MI but rather a week later, simulating real clinical scenarios. ERG is a human protein that enters into the composition of the rapid rectifier potassium channel in myocardial cells, responsible primarily for myocardial repolarization. ERG protein deficiencies have been implicated in inherited and acquired cardiac arrhythmogenesis, leading to sudden cardiac death. In the rabbit model, the ERG protein responds differently to RV pacing under physiological conditions, where its levels increase, ${ }^{8}$ versus disease conditions, such as the aftermath of myocardial infarction, where its levels decrease. ${ }^{4}$ However, the exact mechanism underlying these changes and the role of BIV pacing in restoring ERG levels remain unclear and require further investigation.

The choice of the rabbit model for the present study was driven by numerous considerations, primarily our established experience with this model. ${ }^{4,8,9}$ Our choice of the rabbit in our earlier study of the effect of pacing after MI was dictated by several inherent characteristics of the rabbit heart. ${ }^{10-14}$ The rabbit heart has minimal collateral arteries and no transmural gradient in the collateral blood flow, which leads to MIs that are predictable in size and location. Also, arrhythmic deaths after coronary ligation are less frequent in the rabbit compared with other species, such as dogs. ${ }^{13}$ For these reasons, rabbits have served as a useful animal model for MI provoked by coronary artery ligation. Also, using coronary artery ligation (on the basis of a bifurcation/trifurcation classification) in the rabbit, a reliable chronic heart failure model was developed, ${ }^{15}$ which was the model that we adopted in the current study. The rabbit is also a good model system for electrophysiologic changes. The rabbit cardiac action potential is similar to that of humans, and the underlying channels and currents are also homologous. ${ }^{16-20}$ Moreover, the rabbits' size is large enough to accommodate the implantation of permanent pacemakers designed for humans (but modified to pace at fast rates). For all these reasons, we chose to use the rabbit again, in our present study.

Although human studies have focused on the effect of BIV pacing at the organ level, demonstrating the reduction in $\mathrm{LV}$ dyssynchrony ${ }^{21}$ and mitral regurgitation, ${ }^{22}$ limited knowledge has been accumulated over the past several years as to the cellular and molecular mechanisms involved in the effect of pacing on myocardial function alteration. The present study narrows the gap in knowledge, by documenting changes in the protein expression of genes involved in the ventricular repolarization process. Such an effect may explain the lower incidence of lethal arrhythmias with BIV pacing compared with no pacing, in patients with advanced heart failure. ${ }^{23}$

The present study has a number of limitations. First, the pacing in our rabbit model was initiated 1-2 weeks after coronary ligation, unlike the case in humans, where pacing usually commences years after the MI. However, based on our previous studies, many of the electrical and mechanical remodeling changes occur within this short time frame. It is reasonable therefore, to conclude that BIV pacing works by reversing the adverse remodeling of MI and not only by 
preventing it. Second, in our model, pacing the RV was done epicardially, which is different from the endocardial pacing typically used in humans, in clinical practice. It is unclear whether this difference significantly affects our findings. Third, in our model, the rabbits did not exhibit overt signs of heart failure. This is different from the original indications for BIV pacing. However, more recent data demonstrate benefits from BIV pacing in even mild forms of heart failure. ${ }^{24}$ Lastly, the number of animals included in this study was small thus precluding statistical significance despite the high magnitude of recovery in ERG protein levels (about threefold compared with MI + RV group).

In summary, we present a rabbit model of $\mathrm{MI}$ and pacing that demonstrates recovery of ERG LV protein expression with BIV pacing. This may underlie some of the benefits of cardiac resynchronization therapy post MI.

\section{Acknowledgment}

This study was supported in part by NHLBI grant K08 HL080106-01 (to SS).

\section{Disclosure}

The authors report no conflicts of interest in this work.

\section{References}

1. Linde C, Leclercq C, Rex S, et al. Long-term benefits of biventricular pacing in congestive heart failure: results from the MUltisite STimulation in cardiomyopathy (MUSTIC) study. J Am Coll Cardiol. 2002;40(1): 111-118.

2. Bristow MR, Saxon LA, Boehmer J, et al. Cardiac-resynchronization therapy with or without an implantable defibrillator in advanced chronic heart failure. N Engl J Med. 2004;350(2):2140-2150.

3. Cleland JG, Daubert JC, Erdmann E, et al; Cardiac ResynchronizationHeart Failure (CARE-HF) Study Investigators. The effect of cardiac resynchronization on morbidity and mortality in heart failure. $N$ Engl $J$ Med. 2005;352(15):1539-1549.

4. Saba S, Mathier MA, Mehdi H, et al. Prevention of adverse electrical and mechanical remodeling with biventricular pacing in a rabbit model of myocardial infarction. Heart Rhythm. 2008;5(1):124-130.

5. Petkova-Kirova PS, Gursoy E, Mehdi H, McTiernan CF, London B, Salama G. Electrical remodeling of cardiac myocytes from mice with heart failure due to the overexpression of tumor necrosis factor-alpha. Am J Physiol Heart Circ Physiol. 2006;290(5):H2098-H2107.

6. Mehdi H, Manzi S, Desai P, et al. A functional polymorphism at the transcriptional initiation site in beta2-glycoprotein I (apolipoprotein $\mathrm{H}$ ) associated with reduced gene expression and lower plasma levels of beta2-glycoprotein I. Eur J Biochem. 2003;270(2):230-238.

Research Reports in Clinical Cardiology

\section{Publish your work in this journal}

Research Reports in Clinical Cardiology is an international, peerreviewed, open access journal publishing original research, reports, editorials, reviews and commentaries on all areas of cardiology in the clinic and laboratory. The manuscript management system is completely online and includes a very quick and fair peer-review system.
7. Rose J, Armoundas AA, Tian Y, et al. Molecular correlates of altered expression of potassium currents in failing rabbit myocardium. $\mathrm{Am} \mathrm{J}$ Physiol Heart Circ Physiol. 2005;288(5):H2077-H2087.

8. Saba S, Mehdi H, Mathier MA, Islam MZ, Salama G. London B. Effect of right ventricular versus biventricular pacing on electrical remodeling in the normal heart. Circ Arrhythm Electrophysiol. 2010;3(1):79-87.

9. Saba S, Mathier MA, Mehdi H, et al. Dual-dye optical mapping after myocardial infarction: does the site of ventricular stimulation alter the properties of electrical propagation? J Cardiovasc Electrophysiol. 2008;19(2):197-202.

10. Podesser B, Wollenek G, Seitelberger R, et al. Epicardial branches of coronary arteries and their distribution in the rabbit heart: the rabbit heart as a model of regional ischemia. Anat Rec. 1997;247(4):521-527.

11. Miura T, Downey JM, Ooiwa H, et al. Progression of myocardial infarction in a collateral flow deficient species. Jpn Heart J. 1989;30(5): 695-708.

12. Maxwell MP, Hearse DJ, Yellon DM. Species variation in the coronary collateral circulation during regional myocardial ischaemia: a critical determinant of the rate of evolution and extent of myocardial infarction. Cardiovasc Res. 1987;21(10):737-746.

13. Harken AH, Simson MB, Haselgrove J, Wetstein L, Harden WR 3rd, Barlow CH. Early ischemia after complete coronary ligation in the rabbit, dog, pig, and monkey. Am J Physiol. 1981;241(2):H202-H210.

14. Toyooka T, Kamishiro T, Fumino H, Masaki T, Hosoda S. Rabbit hearts for the critical evaluation of drugs to reduce the size of experimentally produced acute myocardial infarction. Jpn Heart J. 1984;25(4): 623-632.

15. Lee BH, Kim WH, Choi MJ, Rho JR, Kim WG. Chronic heart failure model in rabbits based on the concept of the bifurcation/trifurcation coronary artery branching pattern. Artif Organs. 2002;26(4):360-365.

16. Yehia AR, Shrier A, Lo KC, Guevara MR. Transient outward currents contributes to Wenckebach-like rhythms in isolated rabbit ventricular cells. Am J Physiol. 1997;273(1 Pt 2): H1-H11.

17. Salata JJ, Jurkiewicz NK, Jow B, et al. IK of rabbit ventricle is composed of two currents: evidence for 1Ks. Am J Physiol. 1996;271(6 Pt 2):H2477-H2489.

18. Veldkamp MW, van Ginneken AC, Bouman LN. Single delayed rectifier channels in the membrane of rabbit ventricular myocytes. Circ Res. 1993;72(4):865-878.

19. Fedida G, Giles WR. Regional variations in action potentials and transient outward current in myocytes isolated from rabbit left ventricle. J Physiol. 1991;442:191-209.

20. Suto F, Cahill SA, Wilson GJ, Hamilton RM, Greenwald 1, Gross GJ. A novel rabbit model of variably compensated complete heart block. J Appl Physiol. 2001;92(3):1199-1204.

21. Dohi K, Suffoletto MS, Schwartzman D, Ganz LI, Pinsky MR, Gorcsan J 3rd. Utility of echocardiographic radial strain imaging to quantify left ventricular dyssynchrony and predict acute response to cardiac resynchronization therapy. Am J Cardiol. 2005;96(1):112-116.

22. Kanzaki H, Bazaz R, Schwartzman D, Dohi K, Sade LE, Gorcsan J 3rd. A mechanism for immediate reduction in mitral regurgitation after cardiac resynchronization therapy: insights from mechanical activation strain mapping. J Am Coll Cardiol. 2004;44(8):1619-1625.

23. Barsheshet A, Wang PJ, Moss AJ, et al. Reverse remodeling and the risk of ventricular tachyarrhythmias in the MADIT-CRT (Multicenter Automatic Defibrillator Implantation Trial-Cardiac Resynchronization Therapy). J Am Coll Cardiol. 2011;57(24):2416-2423.

24. Moss AJ, Hall WJ, Cannom DS, et al; MADIT-CRT Trial Investigators. Cardiac-resynchronization therapy for the prevention of heart-failure events. N Engl J Med. 2009;361(14):1329-1338.

\section{Dovepress}

Visit http://www.dovepress.com/testimonials.php to read real quotes from published authors. 